\title{
Stories e Lives no Instagram: comunicação temporária sensível ao contexto
}

\author{
Mateus B. Machado ${ }^{1}$, Caio V. S. Lopes ${ }^{1}$, Ailton Ribeiro ${ }^{2}$, \\ Maria Clara Pestana ${ }^{2}$, Vaninha Vieira ${ }^{1}$ \\ ${ }^{1}$ Departamento de Ciência da Computação (DCC) \\ ${ }^{2}$ Programa de Pós-Graduação em Ciência da Computação - (PGCOMP) \\ Universidade Federal da Bahia (UFBA) \\ \{mateus.machado, caiovsl, ailton.ribeiro, mpestana, vaninha\}@ufba.br
}

\begin{abstract}
Communication on platforms and social networks can be improved when context information is taken into account. In general, in these applications, users need to intervene too much to express ideas and interact with other people. In this sense, this article seeks to investigate communication and proposes a context-sensitive model for Instagram Stories and Lives, features characterized by temporary communication and that can be influenced depending on the context. The design of the new features is discussed in different stages, which involve: an exploratory study with literature review, usage perceptions, survey conducted with 138 users; prototyping and context modeling. The validation of the resources and the analysis of the results, indicates the proposal applicability.
\end{abstract}

Resumo. A comunicação em plataformas e redes sociais pode ser aprimorada quando informações de contexto são consideradas. Em geral, nessas aplicações, os usuários precisam intervir explicitamente e em demasia para expressar ideias e interagir com outras pessoas. Nesse sentido, este artigo busca investigar a comunicação e propor um modelo sensível ao contexto para os Stories e Lives da Instagram, funcionalidades caracterizadas pela comunicação temporária e que pode sofrer influência a depender do contexto. $O$ design dos novos recursos é discutido em diferentes etapas, que envolvem: um estudo exploratório com revisão da literatura, percepções de uso, aplicação de questionário para 138 usuários; prototipação e modelagem de contexto. A validação dos recursos e a análise dos resultados, indicam a aplicabilidade da proposta.

\section{Introdução}

O uso crescente das redes sociais nos últimos anos é influenciado pela disseminação dos dispositivos móveis, que permitem que elas sejam utilizadas a qualquer hora e qualquer lugar. Esse crescimento também pode ser justificado pelo esforço dos desenvolvedores em melhorar a usabilidade e inteligência das interfaces, deixando-as mais intuitivas e naturais [Hui and Sherratt 2016]. As primeiras redes sociais, apesar de terem surgido no cenário da Web e dos computadores fixos, já demonstravam seu potencial comunicativo, ao aproximar pessoas e criar comunidades com milhares de seguidores [Yu et al. 2017]. As redes sociais, então, se aperfeiçoaram e migraram para os dispositivos móveis, cenário que é predominante na atualidade. Esse movimento facilitou a utilização de informações contextuais para gerar adaptações e oferecer serviços mais especializados com base nas 
circunstâncias do usuário. Isso ocorre, pois os dispositivos móveis possuem muitos sensores e conectividade que não existiam nos computadores fixos [Yu et al. 2017]. Uma rede social de destaque e que tira grande proveito desse conceito é o Instagram ${ }^{1}$.

O Instagram se tornou a rede social com maior engajamento no ano de 2019 e possui cerca de 1 (um) bilhão de usuários ativos todos os meses [Mohsin 2020]. É, portanto, uma rede social de grande importância, que se destaca pelo uso do contexto para fornecer serviços mais relevantes aos usuário, como por exemplo, anúncios baseados no histórico de sites acessados na Web e recomendações influenciadas pelo país de origem da pessoa. O contexto, por sua vez, é abordado em [Vieira et al. 2009] como o "conhecimento que está por trás da habilidade de discriminar o que é ou não importante em um dado momento, apoiando indivíduos [...] a compreender certas situações, ações ou eventos".

Dessa forma, este trabalho realiza um estudo sobre a sensibilidade ao contexto em sistemas de comunicação, especialmente no Instagram (plataforma de estudo de caso) e suas funcionalidades de Stories e Lives. Essas funcionalidades têm como característica a comunicação temporária, ou seja, apesar de poderem ser salvos na conta que os publicou, eles ficam disponíveis por um tempo limitado: 24 horas para os Stories e o período da transmissão para as Lives. No trabalho, foram propostos novos recursos sensíveis ao contexto para os Stories e Lives, que são representados em um protótipo (Seção 4) e um modelo de contexto (Seção 4.3). Também foram aplicados dois questionários online com objetivos diferentes. O primeiro questionário teve 138 respostas e foi introduzido na etapa de estudo exploratório para: identificar padrões e tendências de uso, auxiliar na definição dos protótipos e compreender melhor as funcionalidades dos Stories e Lives. $\mathrm{O}$ segundo questionário obteve 33 participações e foi utilizado para avaliar a experiência e a percepção de contexto pelos usuários sobre a proposta construída. Desse modo, o objetivo deste estudo é analisar como a informação de contexto pode influenciar o processo de comunicação temporária na plataforma.

\section{Metodologia}

Este trabalho foi guiado por três etapas metodológicas principais: um estudo exploratório (1), o desenvolvimento de uma proposta (2), e a avaliação da proposta (3). A Figura 1 detalha as etapas metodológicas e cada uma das suas subetapas. As setas roxas determinam o fluxo entre as etapas e as setas com borda determinam o fluxo entre as subetapas. Em algumas situações, foi necessário retornar aos passos anteriores para revisitar conceitos e realizar melhorias. Esse comportamento é representado pelas setas em vermelho. Cada uma dessas etapas são detalhadas a seguir:

1. O estudo exploratório foi realizado com o objetivo de fornecer embasamento teórico para o trabalho, entender o funcionamento do Instagram e levantar as suas características e funcionalidades. A fase inicial envolveu a realização de pesquisa bibliográfica e a criação de testes para serem executados na plataforma. Os testes tiveram o objetivo de gerar percepções pessoais de uso e entender como o contexto estava sendo utilizado. Em conjunto com a pesquisa bibliográfica, os testes foram importantes para definir o foco do estudo nas funcionalidades de Stories e Lives. A fase final do estudo exploratório envolveu uma pesquisa de campo com usuários

\footnotetext{
${ }^{1}$ Uma rede social para compartilhamento de fotos e vídeos: https://www.instagram.com/
} 


\section{Estudo Exploratório (1)}

\section{$\Rightarrow$ ヘิ Definição da Proposta (2)}

(a): Revisão literatura: contexto, sistema sensíveis ao contexto, modelo de contexto e Instagram.

(b): Identificar percepções pessoais de uso.

(c): Pesquisa de campo: questionário online. (a): Construção de protótipos com novos recursos para os Stories e as Lives.

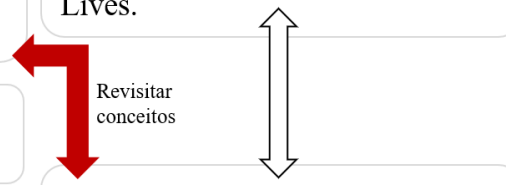

(b): Geração de um modelo de contexto para os Stories e as Lives baseando-se nos protótipos. $\checkmark$ Avaliação da Proposta (3)

(a): Validação das funcionalidades e modelo propostos através de questionário online com usuários do Instagram.

Refinar modelo

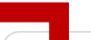

(b): Análise dos resultados obtidos no questionário anterior.

Figura 1. Fluxo das etapas metodológicas.

reais por meio de um questionário online ${ }^{2}$. Essa pesquisa teve caráter tanto qualitativo quanto quantitativo e foi importante para identificar padrões, tendências de uso e definição dos protótipos. O questionário foi construído com o Google Forms $^{3}$ e possui um termo de consentimento livre e esclarecido, que os participantes precisaram aceitar para poder responder. A divulgação do questionário foi feita em listas de e-mails de alunos e professores da Universidade Federal da Bahia (UFBA) e em aplicativos de mensagens instantâneas.

2. A proposta envolveu a elaboração de um modelo de contexto e definição de protótipos interativos, que foram construídos utilizando-se o Figma ${ }^{4}$. Os protótipos foram definidos com base nas informações e resultados obtidos na pesquisa de campo da etapa (1). A seta bidirecional na etapa (2) da Figura 1 indica que o modelo e os protótipos foram produzidos concomitantemente. Inicialmente, foi produzido um rascunho do modelo para auxiliar na construção dos protótipos e posteriormente o modelo foi evoluído.

3. A última etapa do estudo envolveu a realização de uma segunda pesquisa com usuários reais do Instagram com o objetivo de validar e coletar feedbacks sobre a proposta desenvolvida. Essa pesquisa também foi realizada com o uso de um questionário ${ }^{5}$ aplicado de modo online, contendo perguntas abertas e fechadas. O questionário foi construído e divulgado de forma semelhante ao da primeira etapa. A solução proposta foi descrita no questionário através de uma listagem dos recursos implementados, um vídeo demonstrando um uso guiado do protótipo construído e uma versão interativa do protótipo ${ }^{6}$ no Figma. Após a interação com os recursos, foram disponibilizadas perguntas relacionadas para os participantes. Por fim, uma análise foi realizada em cima das respostas obtidas no questionário para avaliar a aceitação e a relevância dos recursos propostos.

\section{Estudo Exploratório}

A primeira etapa do processo de investigação do objeto de estudo envolveu a realização de um estudo exploratório. Este estudo exploratório contemplou as fases necessárias para o

\footnotetext{
${ }^{2}$ Questionário disponível em: http://bit.ly/3rMaoNG

${ }^{3}$ Plataforma de formulários online: https://www.google.com/intl/pt-BR/forms/about/

${ }^{4}$ Uma ferramenta online focada no design de interfaces de usuários: https://www.figma.com/

${ }^{5}$ Questionário disponível em: http://bit.ly/3li9GFa

${ }^{6}$ Protótipo disponível em: https://bit.ly/38aapSO
} 
entendimento mais preciso da plataforma, assim como a identificação de conceitos base, como por exemplo, os conceitos de contexto e sistemas sensíveis ao contexto. Foram documentadas as etapas de revisão de literatura, construção de percepções pessoais a partir do uso empírico da aplicação e pesquisa de campo.

\subsection{Revisão da Literatura}

Uma revisão bibliográfica da literatura foi conduzida para compreender os conceitos abordados nesta pesquisa. A busca por artigos foi realizada nas bases do Google Scholar, Scopus e busca manual em sites de congressos e conferências. Nas seções seguintes, abordar-se-ão os conceitos norteadores da pesquisa sobre sistemas sensíveis ao contexto, a rede social Instagram e modelagem de contexto.

\subsubsection{Sistemas Sensíveis ao Contexto e a Rede Social Instagram}

Muitos sistemas tradicionais e aplicações que existem atualmente, exigem a interferência explícita dos usuários para configurar preferências e fornecer informações acerca da tarefa que desejam executar [Vieira et al. 2009]. Por outro lado, sistemas que possuem a capacidade de adaptar-se às necessidades e às tarefas dos usuários estão sendo desenvolvidos com uma frequência cada vez maior e tendem a se estabelecer como um padrão nos próximos anos. Esta tendência é bem explorada por Vieira, que considera que os "sistemas que exijam demasiada intervenção do usuário para identificar [...] serviços relevantes tendem a perder espaço para aqueles capazes de oferecer serviços mais adaptáveis".

Tais sistemas são denominados sistemas sensíveis ao contexto (CSS, do inglês Context-Sensitive System), e são definidos por Vieira como sistemas complexos que utilizam contexto para dar suporte ao usuário (agente) na execução de determinada tarefa. $\mathrm{O}$ uso do contexto é então percebido pelo usuário na adaptação de interfaces e serviços, em fluxos mais flexíveis e interativos, e na facilidade de usar o sistema para desenvolver a sua tarefa. Para [Dey 2001], os CSSs gerenciam o contexto para "prover informação relevante e/ou serviços para o usuário, sendo que a relevância depende da tarefa do usuário".

O contexto ou as informações contextuais que estão presentes nos CSSs são definidas por Dey como "informações que podem ser utilizadas para caracterizar a situação de uma entidade". Uma entidade, por sua vez, é "uma pessoa, local ou objeto que é considerado relevante na interação entre o usuário e a aplicação [...]". Vieira e colegas, por outro lado, definem contexto a partir da noção de elementos contextuais (CE, do inglês Contextual-Element). Os CEs são qualquer tipo de dado ou informação que podem ser utilizados para definir um contexto. Dessa forma, o conjunto de elementos contextuais que apoiam determinada tarefa, formam o contexto da interação entre um agente e alguma aplicação durante a execução desta tarefa [Vieira et al. 2009].

Nesse sentido, o Instagram é classificado por [Yu et al. 2017] como uma rede social móvel sensível ao contexto CS-MSN (do inglês Context-Sensitive Mobile Social Network). Segundo Yu, redes sociais assim classificadas são uma revolução em relação às redes sociais tradicionais (originalmente utilizadas via Web), pois possibilitam a interação social com alto nível de inteligência a qualquer hora e qualquer lugar. Outras características das CS-MSNs envolvem contexto de alto nível através do contexto pessoal e contexto comunitário. O contexto pessoal é tratado por Yu como informações que caracterizam a situação da pessoa, como afiliação, perfil e localização. Já o contexto comunitário permite entender as atividades, similaridades e relacionamentos da comunidade inteira. 


\subsubsection{Stories e Lives no Instragram: principais usos}

O Instagram foi lançado no ano de 2010, tendo apenas o Feed $^{7}$ de fotos como sua principal funcionalidade. A partir de 2012, o Instagram passou a receber muitas atualizações e ficou disponível em mais de uma loja de aplicativos. Atualmente, dois dos recursos mais populares do Instagram são os Stories e as Lives. Os Stories contam mais de 500 milhões de usuários frequentes [Mohsin 2020] e as lives tiveram grande projeção no ano de 2020 por causa da pandemia do COVID-19. Dados divulgados em [Leskin 2020] mostraram um aumento de 70\% no uso do recurso no início da pandemia em Março de 2020.

Os Stories permitem a comunicação através da postagem de citações, fotos e vídeos na vertical, em combinação com elementos como enquetes, perguntas, música, contagem regressiva, efeitos, emojis, localização, entre outros. Já as Lives são transmissões ao vivo com até duas pessoas aparecendo na tela e contam com chat e comentários em tempo real. Um estudo dirigido pela equipe do [InstagramBusiness 2018] envolvendo usuários de quatro países, revelou que as pessoas vão aos Stories para ver conteúdo ao vivo e por trás das câmeras, e além disso, algumas pessoas concordaram que os Stories facilitam a comunicação com amigos e família.

A pesquisa também evidenciou que, o fato de o conteúdo sumir depois de 24 horas ajuda as pessoas a serem mais autênticas. Essa característica é igualmente explorada em [Pellanda and Streck 2017], que afirmam que os Stories permitem uma visualização quase em tempo real da vida dos usuários e fornecem portanto um contexto da vida das pessoas através das próprias imagens, hashtags, localização entre outros.

\subsubsection{Modelagem de Contexto}

Os modelos de contexto auxiliam a descrever as informações que devem ser consideradas como contexto em determinada aplicação e permitem representar a relação que existe entre essas informações e o APP, ou seja, as adaptações que a aplicação sofre com base na mudança dos valores das informações. Existem diferentes abordagens para modelagem de contexto, que utilizam diferentes técnicas e ferramentas.

Um exemplo de abordagem é o metamodelo de contexto proposto em [Vieira et al. 2009]. Esse metamodelo possui como principais conceitos, a noção de de entidade contextual, elemento contextual (CE, do inglês Contextual-Element), foco e fontes de contexto. A entidade contextual representa um objeto real ou abstrato que está presente nas interações entre o usuário e a aplicação. Os CEs estão associados a alguma entidade e representam uma informação contextual ou propriedade das entidades. $\mathrm{O}$ foco refere-se a uma tarefa que algum agente está desempenhando em determinado momento. Por fim, as fontes de contexto determinam a origem do elemento contextual, que pode ser externo ao sistema, inferido, diretamente informado, entre outros.

Durante o processo de design de um CSS, pode ser difícil identificar os CEs relacionados à situação gerada pela execução da tarefa. Existem algumas técnicas e ferramentas que auxiliam nesse processo, como por exemplo, a técnica que envolve a análise de 4 dimensões básicas: 4Ws, que permite identificar com mais facilidade "quem (who) está fazendo o que (what), em que local (where) e em que momento (when)" [Vieira et al. 2009].

\footnotetext{
${ }^{7} \mathrm{O}$ Feed do Instagram é formado por imagens e vídeos que ocupam uma porção menor da tela e são permanentes na conta dos usuários, a menos que sejam deletados.
} 


\subsection{Percepções de uso: perspectiva dos pesquisadores}

O Instagram é uma rede social recheada de recursos e dispõe de diversas funcionalidades focadas na comunicação, como por exemplo: postagem de fotos, comentários, chat pessoal e em grupo, chamada de vídeo, transmissões ao vivo, entre outros. Esses recursos diversificados fazem do Instagram uma aplicação robusta e complexa. Seria difícil realizar uma análise aprofundada do uso de contexto sobre toda a plataforma e foi, portanto, necessário limitar o escopo da pesquisa e análise dos sistemas de comunicação presentes na plataforma. Para entender o fluxo de funcionamento da aplicação, foram executados testes sobre a mesma. Inicialmente buscou-se analisar superficialmente o sistema do Instagram como um todo, para que posteriormente o foco da pesquisa fosse restringido em apenas algumas funcionalidades.

O primeiro passo envolveu a criação um novo usuário, já que as contas pessoais dos autores já existiam por muito tempo e podiam estar viciadas. Isso faz com que as visualização da adaptação ao contexto por meio da execução de ações arbitrárias pudesse ser prejudicada e dificultar a execução do teste. A criação de um usuário consiste em um cadastro simples com um e-mail ou conectando o novo usuário a uma conta no Facebook. Esta segunda opção reflete em um comportamento que trata contexto, ao indicar as contas dos amigos do Facebook que também estão no Instagram.

Depois da criação da conta, ainda sem seguir nenhum usuário, decidiu-se seguir um usuário da categoria "Esportes" e identificar o comportamento da plataforma. A resposta foi imediata, mesmo seguindo apenas uma conta, a aba "Explorar" foi atualiza e passou a indicar conteúdos relacionados a "Esportes". Isso demonstra, portanto, uma inferência sobre o gosto do usuário através da ação de seguir um perfil. Outro teste realizado envolveu a ação de curtir uma publicação. Seguindo ainda apenas o usuário de "Esportes", testou-se o comportamento de curtir diversas publicações com imagens da categoria "Comida". Desta vez, o comportamento não foi imediato, mas depois de cerca de dez fotos curtidas da mesma categoria, a aba "Explorar" foi atualizada para recomendar publicações de "Esportes" e também de "Comida".

Após diversos testes empíricos na aplicação, foram definidos os recursos que seriam trabalhados. Durante a análise, percebeu-se a subutilização da sensibilidade ao contexto nas ferramentas de Stories e Lives. Estes recursos têm uma característica em comum que os diferencia de alguns outros dentro da plataforma: a temporalidade comunicativa, como já mencionado anteriormente. Essa característica traz possibilidades interessantes quando se considera a sensibilidade ao contexto, o que fez com que os Stories e Lives fossem definidos como foco da pesquisa.

\subsection{Pesquisa de Campo: perspectiva dos usuários}

A pesquisa de campo em formato de questionário online foi realizada para avaliar a experiência e a percepção de contexto pelos usuários quando se comunicam pelo Instagram, além de identificar o sentimento em relação aos Stories e Lives. Foram geradas 138 respostas no total e a maioria dos participantes concentrou-se numa faixa etária de 18 até 25 anos de idade e são estudantes. O perfil jovem dos participantes tem influência nos resultados, já que esse grupo costuma ter mais facilidade de uso nesse tipo de aplicação.

A pesquisa também revelou que $92 \%$ usam o Instagram de forma pessoal, 25,4\% usam de forma comercial e 48,6\% usam para entretenimento. Em relação ao uso diário, 
43,5\% usam por 1 - 3 horas, 30,4\% usam por menos de 1 hora, $21 \%$ usam entre 3 - 5 horas e $5,1 \%$ das pessoas usam o Instagram entre 5 - 8 horas por dia. Em relação ao nível de satisfação com a recomendação de conteúdo, as respostas se concentraram, entre 7 e 8 numa escala de 0 até 10 , sendo 10 uma satisfação maior. Sobre a satisfação geral no aplicativo, as respostas se concentraram, entre 8 e 9 numa escala de 0 até 10.

Em relação à sessão do questionário focada nos Stories, identificou-se que aproximadamente metade dos participantes utilizam os Stories tanto para consumir conteúdo, quanto para produzir conteúdo e a outra metade utiliza apenas para consumo de conteúdo. Os participantes também destacaram a praticidade, interatividade, instantaneidade, naturalidade e a ordem de exibição como fatores que tornam a experiência com os Stories bastante agradável. Também houve interesse em utilizar elementos que podem ser adicionados aos Stories, como localização, música, texto, entre outros, para aprimorar a comunicação indiretamente, apesar da preocupação com questões relacionadas à privacidade. Algumas pessoas destacaram ainda, que esses elementos poderiam ser utilizados para potencializar a interação por meio de diferentes modalidades de comentários.

Referente às Lives, foi identificado que $37.1 \%$ dos participantes utilizam o recurso dos comentários; $62 \%$ não consomem o conteúdo de uma live por completo; e $77.2 \%$ das pessoas relataram que gostariam de ser notificadas de Lives que poderiam gostar. Sobre o que se considera mais agradável na experiência com as Lives, foram obtidas respostas em torno das seguintes questões: maior disseminação de conteúdo e proximidade com o host; comportamento mais espontâneo do host; interação instantânea com o público. Entre as dificuldades relatadas para as Lives, alguns problemas tiveram destaque como: a limitação de recursos de interação com outros usuários presentes; forma ruim de apresentação das perguntas para o host; ausência de opção de ocultar os comentários; disposição das lives no mesmo local que os Stories atrapalha a visualização dos Stories.

\section{Proposta: recursos sensíveis ao contexto para os Stories e Lives}

Os recursos aqui propostos buscam fornecer uma experiência de comunicação melhor ao usuário durante o uso das funcionalidades de Stories e Lives do Instagram. Utiliza-se a sensibilidade ao contexto para tomada de decisões e também organização da informação. Nos tópicos a seguir, são discutidos os protótipos construídos e o funcionamento dos recursos. O protótipo interativo também está disponível no Figma ${ }^{8}$.

\subsection{Comentários e sugestões inteligentes nos Stories}

Baseando-se na possibilidade de utilizar elementos adicionados pelas pessoas nos Stories como informação contextual e nas tendências e motivações de uso identificadas na pesquisa de campo em 3.3, foi proposto o recurso de comentários em grupo e comentários públicos com sugestões e ordenação por relevância. O usuário poderá escolher se quer ativar o recurso no seu Story, ou deixar apenas os comentários privados que já existem, como representado na Figura 2. Nos comentários em grupo, apenas as pessoas marcadas ou identificadas no Story poderão comentar e ver outros comentários. Já nos comentários públicos, qualquer pessoa pode comentar e ver os comentários uns dos outros.

A Figura 3 representa o recurso de sugestões, que traz mais agilidade e engajamento para o usuário. A sugestão é gerada com base em informações presentes no próprio

\footnotetext{
${ }^{8}$ Disponível em: https://bit.ly/38aapSO
} 


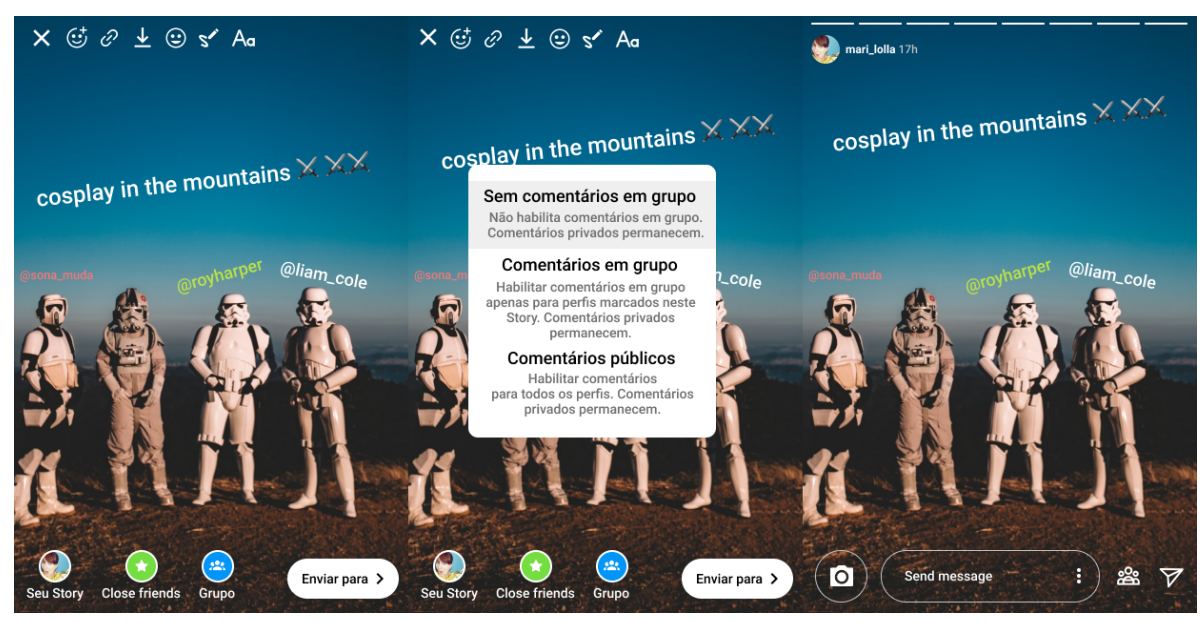

Figura 2. (a) Tela de criação de Story com a opção 'Grupo'; (b) Menu 'Grupo' aberto; (c) Story postado com opção comentário privados e em grupo.

Story, como textos, localização, música, além de informações que o Instagram possui sobre o usuário, como: idade, gênero, momento da vida (estudando, trabalhando, casado) e interesses gerais. Ademais, os comentários aparecem em uma ordem de relevância baseada nas interações e também em informações que o Instagram tem sobre cada usuário. Essas informações são especificadas na Seção 4.3, que trata do modelo de contexto.

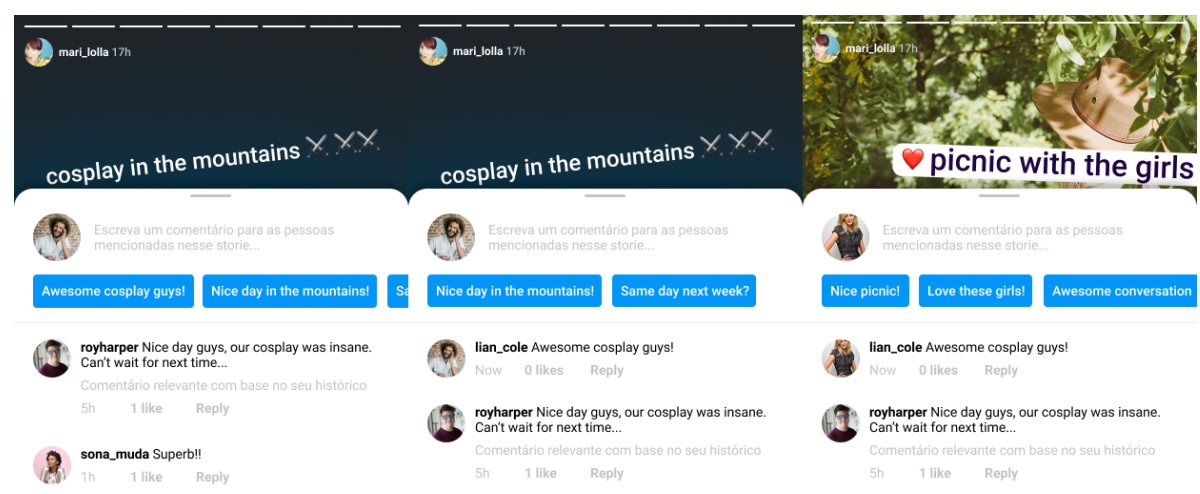

Figura 3. (a) Comentários e sugestões em Story; (b) Sugestão é utilizada e transforma-se em comentário; (c) Outro Story com comentários e sugestões

\subsection{Painel de Lives e Bot}

Para as Lives foram incluídos dois recursos: um painel de Lives e um bot para administração dos comentários durante a realização de uma Live. O painel traz uma reorganização da informação, separando conteúdo em categorias como: Recomendados para você, Amigos, Brasil, Culinária, entre outras, como representado na Figura 4. Para realizar essa divisão, foram levadas em consideração informações de contexto como a localização, a faixa etária, o tempo de permanência do usuário em Lives, o feedback póslive (deu curtida ou não na Live), a atividade (avalia a interação do usuário com a live a partir do número de comentários e curtidas), hashtags (palavras-chave para identificação das temáticas das lives), entre outras informações que são especificadas na Seção 4.3.

A Figura 5 representa o recurso de bot, que é responsável pela administração 
dos comentários em Lives. O usuário pode ativá-lo para que determinadas ações sejam executadas, como: a seleção de comentários relevantes (usando contexto pessoal como critério), advertir usuários de comentários impróprios que violam as diretrizes do Instagram, geração de pergunta única para perguntas frequentes (FAQ) e armazenamento de comentários previamente realizados na Live. Para executar essas ações são levadas em consideração informações de contexto como: o comentário, a partir da análise do seu conteúdo textual para identificar xingamentos ou ofensas e da frequência de utilização de palavras em comentários de outros usuários, e hashtags, que servirão para comparativo na análise de palavras, identificando se o comentário está relacionado ao tema da Live.

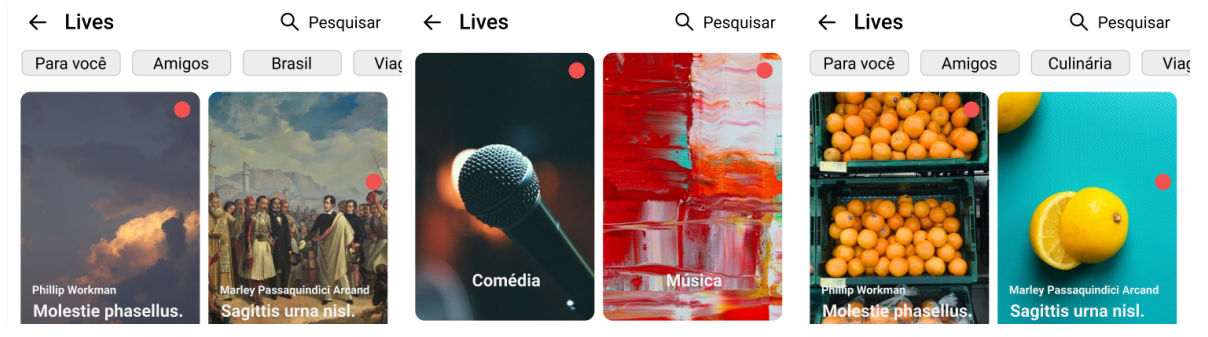

Figura 4. (a) Painel de Lives com suas respectivas categorias; (b) Opções de categorias exibidas ao clicar em 'Pesquisar'; (c) Exemplo da categoria culinária.

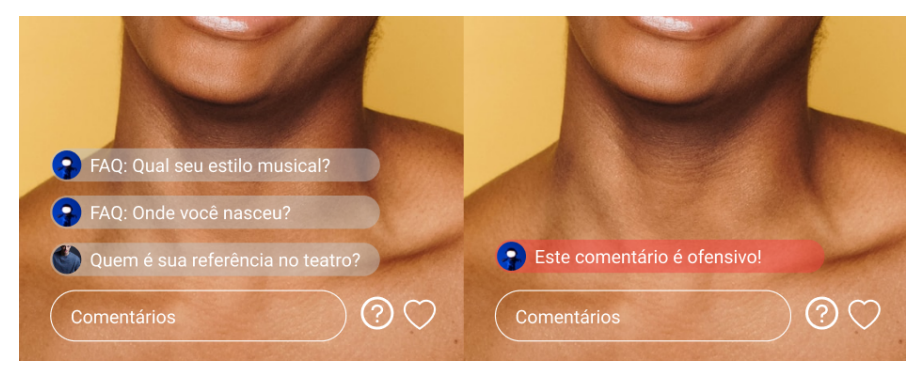

Figura 5. (a) Exibição dos comentários filtrados pelo bot; (b) Visualização do usuário caso insira um comentário que viole as diretrizes do Instagram.

\subsection{Modelo de Contexto para Stories e Lives}

Os modelos de contextos auxiliam os desenvolvedores e projetistas de sistemas a planejar e desenvolver CSSs, pois sistemas deste tipo possuem características diferentes dos sistemas convencionais. Nesse estudo, o modelo de contexto utilizado baseia-se no metamodelo de contexto proposto em [Vieira et al. 2009] e descrito na Seção 3.1.3.

\subsubsection{Stories}

O foco definido para o recurso proposto nos Stories tem como agente uma pessoa qualquer e a tarefa é: interagir via comentários com outros usuários sobre algum Story. A tarefa de criar um Story, como representada no protótipo, é complementar e visa apoiar a tarefa principal definida no foco. A Tabela 1 define o modelo de contexto para a funcionalidade proposta para os Stories. As entidades são representadas por um nome na primeira coluna, e os elementos contextuais são representados pelo seu nome, classificação 4Ws, periodicidade de atualização, fonte e ação ou adaptação causada na plataforma. 
Tabela 1. Modelo $4 \mathrm{Ws}$ para as funcionalidades propostas para os Stories.

\begin{tabular}{|c|c|c|c|c|c|}
\hline \multirow[t]{2}{*}{ Entidade } & \multicolumn{5}{|c|}{ Elemento de Contexto } \\
\hline & Nome & $4 \mathrm{Ws}$ & Altera & Fonte de Contexto & Ação \\
\hline \multirow[t]{6}{*}{ Pessoa } & Hobbies & Who & Pouco & \multirow{4}{*}{$\begin{array}{l}\text { Informado ou importado de } \\
\text { outros sistemas }\end{array}$} & \multirow{4}{*}{$\begin{array}{l}\text { Traz elementos e } \\
\text { características pessoais para } \\
\text { as sugestões }\end{array}$} \\
\hline & Momento da vida & Who & Pouco & & \\
\hline & Gênero & Who & Raro & & \\
\hline & Idade & Who & Pouco & & \\
\hline & $\begin{array}{l}\text { Pessoas } \\
\text { consideradas } \\
\text { próximas }\end{array}$ & Who & Pouco & $\begin{array}{l}\text { Informado, relacionamentos, } \\
\text { histórico de visitas em perfil, } \\
\text { interações em publicações }\end{array}$ & $\begin{array}{l}\text { Determinar relevância do } \\
\text { comentário }\end{array}$ \\
\hline & Localização & Where & Muito & $\begin{array}{l}\text { Obtido via Wi-fi, GPS ou } \\
\text { informado }\end{array}$ & $\begin{array}{l}\text { Fornecer ao Story a } \\
\text { localização da pessoa }\end{array}$ \\
\hline \multirow[t]{6}{*}{ Story } & Tag Localização & Where & Nunca & $\begin{array}{l}\text { Obtido via Wi-fi, GPS ou } \\
\text { informado }\end{array}$ & $\begin{array}{l}\text { Enriquece a sugestão com o } \\
\text { local onde Story foi criado }\end{array}$ \\
\hline & Marcar pessoa & What & Pouco & Informado pela pessoa & Habilitar comentários em grupo \\
\hline & $\begin{array}{l}\text { Texto, como frases, } \\
\text { perguntas e respostas }\end{array}$ & Who & Nunca & Informado pela pessoa & $\begin{array}{l}\text { Enriquece a sugestão gerada } \\
\text { para o comentário }\end{array}$ \\
\hline & Emojis & Who & Nunca & Informado pela pessoa & $\begin{array}{l}\text { Fornece noção de sentimento } \\
\text { para a sugestão }\end{array}$ \\
\hline & Imagem & Where & Nunca & $\begin{array}{l}\text { Retratado pela câmera ou pasta } \\
\text { de fotos do usuário }\end{array}$ & \multirow{2}{*}{$\begin{array}{l}\text { Traz elementos do ambiente } \\
\text { e pessoas envolvidas para as } \\
\text { sugestões }\end{array}$} \\
\hline & Vídeo & Where & Nunca & $\begin{array}{l}\text { Retratado pela câmera ou pasta } \\
\text { de fotos do usuário }\end{array}$ & \\
\hline \multirow[t]{3}{*}{ Comentário } & Data de publicação & When & Nunca & Registrado no servidor & $\begin{array}{l}\text { Determinar relevância do } \\
\text { comentário }\end{array}$ \\
\hline & Responder & What & Pouco & Informado pela pessoa & \multirow{2}{*}{$\begin{array}{l}\text { Maior relevância para } \\
\text { comentário }\end{array}$} \\
\hline & Dar likes & What & Pouco & Informado pela pessoa & \\
\hline Calendário & Data comemorativa & When & Nunca & Agenda e serviço de datas & $\begin{array}{l}\text { Trazer elementos de datas } \\
\text { comemorativas para sugestões }\end{array}$ \\
\hline
\end{tabular}

Na Tabela 1, o elemento Hobbies pode ser utilizado, por exemplo, em um Story que retrata uma tarde de jogos de uma pessoa com os amigos. Sabendo que um dos hobbies da pessoa é jogar com amigos, uma sugestão de comentário pode ser: "Ótima tarde, jogar é um dos meus passatempos favoritos". Nesse caso, uma característica pessoal está presente na sugestão, deixando-a com mais personalidade e precisão. Vários outros elementos de contexto detalhados na Tabela 1 têm uma influência semelhante nas sugestões.

\subsubsection{Lives}

Para os recursos propostos nas Lives, o foco tem uma pessoa qualquer como agente e a tarefa é: assistir uma live e interagir via comentários durante a realização da mesma. A tarefa do painel de lives, como representada no protótipo, é complementar e funciona 
apenas como um passo para executar a tarefa principal definida. As entidades e elementos contextuais são apresentados na Tabela 2, que segue a mesma estrutura descrita para a Tabela 1, exceto pela classificação $5 \mathrm{~W} 1 \mathrm{H}$, uma extensão da técnica $4 \mathrm{Ws}$, que considera $\mathrm{o}$ motivo (why) e a maneira (how).

Tabela 2. Modelo 5W1H para as funcionalidades propostas para as Lives.

\begin{tabular}{|c|c|c|c|c|c|}
\hline \multirow[t]{2}{*}{ Entidade } & \multicolumn{5}{|c|}{ Elemento de Contexto } \\
\hline & Nome & $5 \mathrm{~W} 1 \mathrm{H}$ & Altera & Fonte de Contexto & Ação \\
\hline \multirow[t]{4}{*}{ Pessoa } & $\begin{array}{l}\text { Usuários com } \\
\text { interesses convergentes }\end{array}$ & Who & Muito & $\begin{array}{l}\text { Inferência decorrente de } \\
\text { Filtragem Colaborativa }\end{array}$ & $\begin{array}{l}\text { Recomenda Lives assistidas } \\
\text { pelo usuário X para o usuário } \mathrm{Y}\end{array}$ \\
\hline & Localização & Where & Muito & $\begin{array}{l}\text { Obtido via Wi-fi, GPS ou } \\
\text { informado }\end{array}$ & $\begin{array}{l}\text { Recomenda Lives acontecendo } \\
\text { na localização atual }\end{array}$ \\
\hline & $\begin{array}{l}\text { Tempo de } \\
\text { permanência }\end{array}$ & What & Muito & Histórico de acessos & $\begin{array}{l}\text { Recomenda Lives com temas } \\
\text { semelhantes aquelas que a taxa } \\
\text { de permanência é maior }\end{array}$ \\
\hline & Interação do usuário & What & Muito & Histórico de acessos & $\begin{array}{l}\text { Recomenda Lives com temas } \\
\text { semelhantes aquelas que a taxa } \\
\text { de interatividade é maior }\end{array}$ \\
\hline Calendário & Data comemorativa & When & Pouco & Agenda e serviço de datas & Recomenda Lives sazonais \\
\hline \multirow[t]{3}{*}{ Comentário } & $\begin{array}{l}\text { Conformidade com as } \\
\text { diretrizes da aplicação }\end{array}$ & How & Pouco & \multirow{3}{*}{$\begin{array}{l}\text { Inferência decorrente de } \\
\text { processamento de } \\
\text { linguagem natural }\end{array}$} & $\begin{array}{l}\text { Bloquear comentário e } \\
\text { alertar usuário }\end{array}$ \\
\hline & Frequência & How & Muito & & $\begin{array}{l}\text { Gera comentário único a partir } \\
\text { dos comentários frequentes }\end{array}$ \\
\hline & Relevância & How & Muito & & Destaca comentário relevante \\
\hline
\end{tabular}

\section{Avaliação e Resultados}

Após o processo de prototipação e modelagem, foi realizada outra pesquisa de campo em formato de questionário online para avaliar a experiência e a percepção de contexto pelos usuários sobre as propostas construídas. Houveram 33 participantes no total, dos quais $51,5 \%$ também responderam o primeiro questionário.

Os comentários públicos foram avaliados em relação a sua utilidade. Em uma escala de 0 até 10,42,5\% dos participantes deram notas situadas entre 7 e 10 e 30,3\% deram notas entre 4 e 6 . Os comentários em grupo foram bem aceitos, e 45,4\% das notas se concentraram entre 8 e 10. A utilidade de sugestões em comentários foi avaliada de forma equilibrada pelos participantes. Isso pode indicar que algumas pessoas não acreditam que as sugestões podem ser apropriadas o suficiente a ponto de serem utilizadas de fato. Porém, ao mencionar que as sugestões são influenciadas pela informação contextual presente no Story, a aceitação foi maior, com 54,5\% das notas situadas entre 8 e 10 . Sobre os elementos mais relevantes para gerar sugestões, os usuários consideram as pessoas marcadas, localização e perguntas e respostas inseridas no Story como as informações mais importantes. Houve também uma aceitação muito boa em relação à ordem de exibição dos comentários com base na proximidade que possuem com a pessoa.

Referente às Lives, verificou-se que $90.9 \%$ dos participantes consideram que as recomendações e a divisão de categorias das Lives faz sentido. Com relação a utilização 
do recurso do bot, $33.3 \%$ respondeu que usaria se estivesse produzindo uma live, $27.3 \%$ se estivesse consumindo uma live e $30.3 \%$ usaria em ambos os casos. Por fim, sobre os recursos elencados como mais relevantes, $50 \%$ marcaram o aviso de comentário impróprio como relevante e $46.9 \%$ marcaram a seleção de perguntas frequentes (FAQ).

\section{Conclusão e Trabalhos Futuros}

Neste estudo, foi apresentado um modelo de contexto para novas funcionalidades de comentário com sugestões inteligentes nos Stories e para os painéis e o bot para as Lives. Os recursos foram demonstrados em um protótipo interativo e utilizaram as informações contextuais para aprimorar a experiência de comunicação entre as pessoas. A etapa de avaliação mostrou que a proposta desenvolvida foi bem aceita pelos participantes e as interfaces construídas foram consideradas fáceis de se utilizar e intuitivas, características que são bastante importantes nos sistemas da computação ubíqua. Pode-se destacar ainda a percepção pelos participantes de uma comunicação mais eficiente e facilitada.

Para trabalhos futuros, é importante analisar como a rotatividade dos Stories vai influenciar nos comentários públicos e em grupo. Em muitas ocasiões, as pessoas postam muitos Stories sobre um mesmo conteúdo, em um mesmo local, e com os mesmos usuários relacionadas, ou seja, um mesmo contexto. Pode-se tornar desestimulante produzir comentários em cada um desses Stories, já que eles retratam situações semelhantes. Outra questão envolve o estudo do impacto que o Bot irá trazer para Lives, pois um dos fatores que gera engajamento em transmissões ao vivo é a possibilidade de comentar livremente. Uma limitação do trabalho está no fato de que os recursos foram projetados para uma aplicação proprietária e a princípio não serão implementados por quem desenvolve o aplicativo, o que dificulta uma validação com maior precisão dos recursos propostos.

\section{Referências}

[Dey 2001] Dey, A. K. (2001). Understanding and using context. Personal and ubiquitous computing, 5(1):4-7.

[Hui and Sherratt 2016] Hui, T. K. L. and Sherratt, R. S. (2016). Towards disappearing user interfaces for ubiquitous computing: human enhancement from sixth sense to super senses. Journal of Ambient Intelligence and Humanized Computing, 8:449-465.

[InstagramBusiness 2018] InstagramBusiness (2018). How do people perceive and use instagram stories and feed? https://bit.ly/3oMhekA, December.

[Leskin 2020] Leskin, P. (2020). Instagram live usage jumped 70\% last month [..]. https://bit.ly/38RIXMB, December.

[Mohsin 2020] Mohsin, M. (2020). 10 estatísticas do instagram que todo empreendedor precisa conhecer em 2021. https://www.oberlo.com.br/blog/estatisticas-instagram.

[Pellanda and Streck 2017] Pellanda, E. and Streck, M. (2017). Instagram como interface da comunicação móvel e ubíqua. Sessões do Imaginário, 22(37):10-19.

[Vieira et al. 2009] Vieira, V., Tedesco, P., and Salgado, A. C. (2009). Modelos e processos para o desenvolvimento de sistemas sensíveis ao contexto. JAI, pages 381-431.

[Yu et al. 2017] Yu, Z., Zhang, D., Wang, Z., Guo, B., Roussaki, I., Doolin, K., and Claffey, E. (2017). Toward context-aware mobile social networks. IEEE CM, 55(10):168-175. 